\title{
Coagulopatia em gestantes com COVID-19
}

\author{
Coagulopathy in pregnant women with COVID-19 \\ Coagulopatía en mujeres embarazadas com COVID-19
}

Recebido: 19/10/2021 | Revisado: 30/10/2021 | Aceito: 23/11/2021 | Publicado: 04/12/2021

Júlia de Lima Santos

ORCID: https://orcid.org/0000-0002-1239-0443 Universidade do Estado de Minas Gerais, Brasil E-mail: julialima_123@outlook.com.br

Thatiany Santos Araújo

ORCID: https://orcid.org/0000-0002-8995-2758 Universidade do Estado de Minas Gerais, Brasil E-mail: thatiany_araujo@hotmail.com.br

João Junior Scapin Telis

ORCID: https://orcid.org/0000-0003-0989-1922 Universidade do Estado de Minas Gerais, Brasil E-mail: joaobiomeduemg@gmail.com

Mikael Santos Araújo

ORCID: https://orcid.org/0000-0001-6147-9703 Universidade do Estado de Minas Gerais, Brasil E-mail: mikaelme91@gmail.com Jennifer Soares de Almeida ORCID: https://orcid.org/0000-0003-0989-1922 Universidade do Estado de Minas Gerais, Brasil E-mail: jennifer.almeida.05@gmail.com

Thalita Grazielly Santos ORCID: https://orcid.org/0000-0001-5292-8343 Universidade do Estado de Minas Gerais, Brasil E-mail: thalita.santos@urmg.br

Nicole Blanco Bernardes ORCID: https://orcid.org/0000-0003-3548-0553 Universidade do Estado de Minas Gerais, Brasil E-mail: nicole.bernardes@uemg.br

\section{Resumo}

A atual pandemia da COVID-19, causada pelo novo coronavírus SARSCoV-2, teve rápida disseminação e resultou em inúmeros casos fatais. A coagulopatia é uma das principais complicações dessa doença, sendo possível a ocorrência em qualquer paciente infectado pelo vírus; um dos grupos de risco, as pacientes obstétricas, requer maior atenção, devido às alterações coagulativas e hemostáticas já esperadas neste grupo. O objetivo da revisão foi realizar um levantamento bibliográfico de artigos que abordam a relação entre hipercoagulopatia e gestantes infectadas pelo vírus SARS-COV-2. A pesquisa foi efetuada nas bases de dados Google Acadêmico, Biblioteca Virtual em Saúde (BVS), Scientific Electronic Library Online (Scielo) e PubMed. Foram utilizados os seguintes descritores "gestantes", "coagulopatia", "doença sanguínea", "COVID-19", "alterações hematológicas". Alterações de coagulação ocorrem em pacientes gestantes ou não infectadas pelo SARS-COV-2. As gestantes são um grupo mais susceptível à agravos, devido ao processo de hipercoagulabilidade que já ocorre naturalmente no período gestacional. Faz-se necessário mais estudos que evidenciem essa associação, a fim de prover os cuidados e atendimento adequados a tais pacientes.

Palavras-chave: Gestantes; Coagulopatia; Doença sanguínea; Alterações hematológicas; COVID-19.

\begin{abstract}
The current COVID-19 pandemic caused by the new SARSCoV-2 coronavirus, spread rapidly and resulted in numerous fatal cases. Coagulopathy is one of the main complications of this disease, and it can occur in any patient infected by the virus; one of the risk groups, obstetric patients, requires more attention, due to coagulative and hemostatic changes expected in this group. The purpose of the review was to conduct a literature review of articles that address the relationship between hypercoagulopathy and pregnant women infected by the SARS-COV-2 virus. The search was performed in Google Scholar, Virtual Health Library (BVS), Scientific Electronic Library Online (Scielo) and PubMed databases. The following descriptors were used "pregnant women", "coagulopathy", "blood disease", "COVID-19", "haematological alterations". Bleeding disorders occur in patients who are pregnant or not infected by the SARS-COV-2. Pregnant women are a group more susceptible to aggravations, due to the hypercoagulability process that already occurs naturally in the gestational period. More studies are needed to show this association, in order to provide adequate care for such patients.
\end{abstract}

Keywords: Pregnant; Pregnant woman; Coagulopathy; Blood disease; COVID-19. 


\section{Resumen}

La pandemia actual de COVID-19, causada por el nuevo coronavirus SARSCoV-2, se propagó rápidamente y resultó en numerosos casos fatales. La coagulopatía es una de las principales complicaciones de esta enfermedad y puede presentarse en cualquier paciente infectado por el virus; Uno de los grupos de riesgo, las pacientes obstétricas, requiere mayor atención, debido a los cambios coagulativos y hemostáticos esperados en este grupo. El objetivo de la revisión fue realizar un relevamiento bibliográfico de artículos que abordan la relación entre la hipercoagulopatía y las embarazadas infectadas por el virus SARS-COV-2. La búsqueda se realizó en las bases de datos Google Academic, Virtual Health Library (BVS), Scientific Electronic Library Online (Scielo) y PubMed. Se utilizaron los siguientes descriptores "mujeres embarazadas", "coagulopatía", "enfermedad de la sangre", "COVID-19", "alteraciones hematológicas". Las alteraciones de la coagulación ocurren en pacientes embarazadas o no infectadas con SARSCOV-2. Las mujeres embarazadas son un grupo más susceptible a las lesiones, debido al proceso de hipercoagulabilidad que ya ocurre de forma natural durante el embarazo. Se necesitan más estudios para demostrar esta asociación, con el fin de proporcionar una atención y un cuidado adecuados a estos pacientes.

Palabras clave: Mujeres embarazadas; Coagulopatía; Enfermedad de la sangure; Cambios hematológicos; COVID19.

\section{Introdução}

A COVID-19, também chamada de Síndrome Respiratória Aguda Grave do coronavírus 2 (Sars-Cov-2), foi identificada na China, tendo seu primeiro caso relatado no final de 2019, na cidade de Wuhan. No dia 30 de janeiro de 2020, a doença foi declarada pela Organização Mundial da Saúde (OMS) uma emergência de Saúde e Pública de relevância mundial, e, em março do mesmo ano, declarou-se a COVID-19 como uma pandemia mundial.

O vírus da COVID-19 (SARS-COV-2) é um vírus de RNA, ou seja, seu material genético consiste em uma molécula única de RNA. Vale ressaltar também que nele, encontram-se cerca de 29 proteínas virais. Dentre elas, cabe citar a glicoproteína de pico cuja função é permitir a entrada do vírus na célula através de sua ligação aos receptores de membrana e a proteína do nucleocapsídeo que regula a replicação viral (Uzunian, 2020).

Foi relatado em todo o mundo que pacientes internados com a doença supracitada, estão desenvolvendo anormalidades laboratoriais, dentre elas, merecem destaque, a hipercoagulabilidade, estase venosa, turbulência e eventos tromboembólicos (Iba et al., 2020).

Apesar dos resultados dos exames laboratoriais em pacientes obstétricas se assemelharem com os resultados da população em geral, percebe-se que a resposta inflamatória neste primeiro grupo, principalmente quando infectados por COVID-19, se mostra mais grave. Nesse viés, durante surtos virais anteriores, a comorbidade e mortalidade materna foi, significativamente, alta. Ademais, ressalta-se que em pacientes obstétricas, a interpretação dos testes de coagulação pode ser mais desafiadora visto que a gravidez em si já apresenta aumento na suscetibilidade de doenças por coagulação (Benhamou, Keita, Ducloy-Bouthors, 2020).

Desse modo, devido às proporções tomadas pela pandemia e o limitado conhecimento a respeito da forma como o SARS-COV-2 relaciona-se com alterações de coagulação em mulheres grávidas, faz-se necessário o estudo deste tema. Isso porque há poucos registros publicados, até então. Portanto, o aprimoramento acerca do assunto é permitido e uma posterior contribuição com a comunidade científica é realizada.

Devido ao panorama atual da pandemia, a covid-19 destaca-se como um assunto relevante. Apesar de já muito discutido, há poucos estudos que relaciona o vírus SARS-COV-2 com coagulopatia em gestantes. A gravidez é, geralmente, considerada um estado de alto risco no contexto de condições infecciosas, pois as alterações imunológicas promovidas por ela podem aumentar a suscetibilidade a patógenos e suas complicações (Seyda et al., 2020). Nesse contexto, a complexa interação entre a resposta imune à infecção e a ativação de vias de coagulação resulta em um estado pró-coagulante em pacientes afetados por COVID-19 (Seyda et al., 2020). Assim, é necessário que mais informações sejam obtidas para facilitar o prognóstico médico. 
Os objetivos consistem em realizar uma revisão bibliográfica a fim de identificar alterações na coagulação de gestantes infectadas pelo vírus SARS-COV-2. Especificamente, espera-se aprimorar os conhecimentos sobre o vírus e alterações na coagulação em gestantes.

\section{Metodologia}

O estudo trata-se de uma revisão narrativa realizada através do levantamento bibliográfico de artigos; para tal, utilizaram-se os seguintes descritores: "gestantes", "coagulopatia", "doença sanguínea", "COVID-19", "alterações hematológicas", "pregnant", "pregnant woman", "coagulopathy", "blood disease" associados ao operador boleano AND. Foram empregados como bases de dados a Biblioteca Virtual em Saúde (BVS), Scientific Electronic Library Online (Scielo) e PubMed. Os critérios de inclusão foram: artigos que associam a COVID-19 com coagulopatia em gestantes, escritos em português ou inglês e de acesso gratuito e na íntegra. Excluíram-se trabalhos de conclusão de curso (TCC), monografias, dissertações e artigos duplicados.

\section{Resultados e Discussão}

De acordo com os dados e informações, divulgados em todo o mundo, os pacientes hospitalizados infectados pelo vírus da COVID-19 estão, frequentemente, desenvolvendo alterações hematológicas correspondentes a hipercoagulabilidade e, clinicamente, a eventos tromboembólicos (Iba et al., 2020). Ranucci et al (2020) relataram como principais alterações, associadas aos distúrbios de coagulação, o aumento de D-dímeros e dos níveis de fibrinogênio. Panigada et al (2020) observaram resultados semelhantes, incluindo o aumento dos níveis de fibrinogênio, da ativação plaquetária e das variáveis viscoelásticas. Tang et al (2020) também relataram aumento de D-dímero e produtos de degradação da fibrina associados com tempo de protrombina (TP) e tempo de tromboplastina parcial ativada (TTPA) ligeiramente a moderadamente aumentados. Uma ampla variedade de sintomas pode ser apresentada nos relatos de casos de COVID-19. Assim, os pacientes ficam predispostos às condições patológicas na circulação venosa e arterial, como a trombose. Os aumentos marcantes na fibrina/ fibrinogênio são sinais iniciais de coagulopatia por COVID-19 (Gunduz, 2021).

Ji et al (2020) apontam a elevação de D-dímero como uma consequência da plasmina ativada e do aumento da atividade fibrinolítica. Iba et al (2020) associam o aumento dos níveis de fibrinogênio e da geração de trombina à cascata de coagulação, iniciada em infecções virais, como forma de defesa do hospedeiro contra os patógenos. Benhamou, Keita e Ducloy-Bouthors (2020) sugerem que a infecção por COVID-19 causa uma resposta inflamatória exagerada, comumente chamada de "tempestade de citocinas". Assim, conclui-se que a coagulopatia é uma característica frequente na infecção por SARS-CoV-2 e os níveis de D-dímero elevados são o achado mais comum (Iba et al., 2020). Ressalta-se que alterações da coagulação podem ter um papel patogênico direto, principalmente por causar trombos (macro e micro) em vários órgãos, reduzindo o fluxo sanguíneo nos capilares e agravando a lesão local (Benhamou, Keita, Ducloy-Bouthors, 2020).

Em surtos virais anteriores, a mortalidade e morbidade materna de gestantes foi especialmente alta, entretanto os quadros clínicos de pacientes obstétricas, com infecção por COVID-19, não foram piores do que os da população em geral, mas a resposta inflamatória nessas pacientes parece ser mais grave (Benhamou, Keita, Ducloy-Bouthors, 2020).Ronnje et al (2020) sugerem que as respostas imunológicas induzidas pela gravidez podem exagerar o curso da infecção por COVID-19 e, por ser um estado de hipercoagulação, a gestação está associada ao alto risco de complicações trombóticas maternas. De acordo com Prabhu et al (2020), 78,6\% das mulheres gravidas com COVID-19 eram assintomáticas e mesmo nesse grupo, foi afirmado que a presença do vírus SARS-Cov-2 aumenta significativamente a chance de se desenvolver trombose. Em grávidas sem COVID-19 a taxa do risco absoluto de complicações tromboembólicas é de $0,1 \%$, já quando há associação de grávidas com infecção por COVID-19, essa taxa aumenta para 0,98\% e 0,28\% para as complicações homeostáticas e tromboembólicas, 
respectivamente. Nesse sentido, foram encontrados nas placentas de 48\% das grávidas diagnosticadas com COVID-19, vestígios de eventos tromboembólicos nos vasos fetais. Entretanto, o trabalho de Rodrigues, Baía, Domingues e Barros (2020) apresenta dados que não relacionam gestantes portadoras de COVID-19 com coagulopatias. Desse modo, mulheres grávidas infectadas pelo SARS-Cov-2 possuem os mesmos riscos de mulheres não infectadas. No entanto, relata que a forma grave da doença pode ser mais recorrente em pacientes portadoras de comorbidades como obesidade, asma e diabetes.

Segundo Benhamou, Keita e Ducloy-Bouthors (2020), a interpretação dos testes de coagulação e possíveis anormalidades, em gestantes infectadas pelo coronavírus, pode ser muito desafiador, pois os resultados são confundidos por alterações de coagulação normalmente induzidas pela gravidez. JE Mongula et al (2020), afirmam que nos pacientes portadores da COVID-19 não grávidas, a deposição de fibrina ocorre no pulmão gerando grave sintomatologia; já nas gestantes portadoras de tal patologia, essa deposição pode ocorrer na placenta, onde a paciente poderá desenvolver coagulopatia associada à COVID-19.

Segundo Makatsariya et al (2020), uma mulher grávida passa por diversas transformações durante a gestação, principalmente quando se remete ao sistema cardiovascular, onde temos mudanças como hipercoagulação fisiológica, retardamento do fluxo venoso, aumento da capacitância venosa, entre outras, sendo todas importantes para precaver hemorragia no parto. No entanto, tais mudanças favorecem o risco de tromboembolismo venoso em até 5 a 6 vezes mais que o normal, e é por isso que existe o sistema compensatório que através de mecanismos particulares irão manter o equilíbrio, isso quando se fala de uma gravidez normal e saudável.

Os principais distúrbios de coagulação observados ao longo do curso normal da gestação são: aumento da concentração de fibrinogênio e dos valores de D-dímero; queda da contagem de plaquetas; encurtamento do tempo de tromboplastina parcial ativada (TTPA) e do tempo de protrombina (TP), devido ao acentuado aumento da concentração plasmática da maioria dos fatores de coagulação e redução do nível de antitrombina III (Ronnje et al., 2020; Benhamou, Keita, Ducloy-Bouthors, 2020).

Porém, ressalta-se que a coagulopatia associada à COVID-19 não foi totalmente descrita no momento de apresentação no início da pandemia e a literatura sugere, para a população não grávida, um possível componente pró-trombogênico associado à COVID-19. Desse modo, essa doença se relaciona com distúrbios de coagulação tanto para gestantes, quanto para a população geral (Makatsariya et al., 2020).

Segundo Cubas et al (2020), é considerado a vulnerabilidade das mulheres grávidas quando infectadas pelo SARSCov-2. Nesse estudo, foram relatadas 3 mortes por COVID-19 de mulheres grávidas, sendo uma delas causada por acidente vascular cerebral que é um episódio intimamente atrelado a formação de trombos. Desse modo, é possível inferir que a COVID-19 está relacionada com a coagulopatia em gestantes. Além disso, foi estabelecida uma média de 33,3\% das gestantes infectadas pelo vírus da COVID-19, apresentarem sintomas como eclampsia e pneumonia, sendo o primeiro decorrente de eventos tromboembólicos.

O trabalho de George Nesr et al (2020) foi realizado com pacientes gestantes e portadoras de trombocitopenia imune (PTI) com infecções leves por Covid-19. No estudo nota-se que a infecção por Covid-19 foi um fator para o agravamento da coagulopatia em questão.

O estudo realizado por Kadir et al (2020) destacou questões hemostáticas específicas durante a gravidez. Foram construídas recomendações preliminares para ajudar no cuidado de grávidas afetadas por COVID-19 com coagulopatia ou complicações trombóticas. O estudo revelou que portadoras da COVID-19 demonstram uma taxa de letalidade comparável entre gestantes e não gestantes, entretanto grávidas com tempo de gestação avançada tem mais propensão a adquirir doenças graves. 


\section{Conclusão}

A partir dos resultados encontrados e analisados nessa revisão narrativa, pode-se concluir que as alterações tromboembólicas e hematológicas estão nitidamente presentes em grávidas, pessoas portadoras de COVID-19 e em pacientes com coagulopatias. Portanto, com a infecção por COVID-19, podem ocorrer alterações adicionais de coagulação, as quais podem acentuar a gravidade da doença e existem preocupações consideráveis quanto ao risco de complicações trombóticas e coagulopatia em pacientes obstétricas, embora ainda faltem dados robustos. A infecção pelo SARS-Cov-2 é um agravante ao processo de hipercoagulabilidade, que já ocorre naturalmente durante o período gestacional. As mulheres grávidas devem ser consideradas um grupo vulnerável na população e sua exposição ao COVID-19 deve ser evitada.

Tal discussão confirma a necessidade de novos trabalhos, experiências e fornecimento de dados voltados para esta área, através de pesquisas de campo, visto que ainda não há evidências concretas a cerca da COVID-19 como causa única para o agravamento de coagulopatias em gestantes.

\section{Referências}

Assunção, P., Costa, E \& Dantas, E. (2017). Perfil epidemiológico das coagulopatias hereditárias no estado de alagoas no período de 2011 - 2015. Centro Universitário Tiradentes - UNIT.

Barca, D. et al. (2010). Hemovida web coagulopatias: um relato de seu processo de desenvolvimento e implementação. Hemovida web coagulopatias, 434444.

Benhamou, D., Keita, H. \& Ducloy-bouthors, A. S. (2020). Obstetric Anaesthesia and Critical Care Club Working Group (2020). Coagulation changes and thromboembolic risk in COVID-19 obstetric patients. Anaesthesia, critical care \& pain medicine, 39 (3), 351-353. doi: 10.1016/j.accpm.2020.05.003.

Cubas, J. et al. (2020). Maternal mortality in pregnant woman with positive sars-cov-2 antibodies and severe preeclampsia. report of cases. Rev Peru Obstet. 2020; 66, 1-6.

Dashraath, P. et al. (2020). Coronavírus disease 2019 (COVID-19) pandemic and pregnancy. Am J Obstet Gynecol.

Geng, YJ et al. (2020). Pathophysiological characteristics and therapeutic approaches for pulmonary injury and cardiovascular complications of coronavirus disease 2019. Cardiovascular Pathology. 47..

Giannis, D., Ziogas, I. \& Gianni, P. (2020) Coagulation disorders in coronavirus infected patients: COVID-19, SARS-CoV-1, MERS-CoV and lessons from the past. Journal of Clinical Virology.

Gorziza, R. (2020). Avaliação laboratorial de coagulopatias e trombpfilias https://www.lume.ufrgs.br/bitstream/handle/10183/186447/Resumo_12373.pdf?sequence=l.

Gunduz, Z. (2021). Venous sinus thrombosis during COVID-19 infection in pregnancy: a case report. São Paulo Med J. doi: https://doi.org/10.1590/15163180.2020.0659.R1.08122020.

Iba, T. et al. (2020). Coagulopathy in COVID-19. J Thromb Haemost; 18, 2103-2109. doi: https://doi.org/10.1111/jth.14975,

Iba, T. et al. (2020). Coagulopathy of Coronavirus Disease 2019. Critical care medicine, 48, (9), 1358-1364. doi: https://doi.org/10.1097/CCM.0000000000004458.

Javanmard, S., Heshmat-ghahdarijani, K. \& Vaseghi, G. (2020). Angiotensin-converting-enzyme inhibitors (ACE inhibitors) and angiotensin II receptor blocker (ARB) use in COVID-19 prevention or treatment: A paradox. Infection Control \& Hospital Epidemiology, 1-2.

Kadir, R. et al. (2020). Covid-19 coagulopathy in pregnancy: critical review, preliminary recommendations, and isth registry-communication from the isth ssc for women's health. International Society on Thrombosis and Haemostasis. doi: doi: 10.1111/JTH.15072.

Kang, Y. et al. (2020). Cardiovascular manifestations and treatment considerations in COVID-19. Heart, 106(15), $1132-114$.

Koumoutsea, E. et al. (2020). COVID-19 and acute coagulopathy in pregnancy. J Thromb Haemost, 18, 1648- 1652. doi: https://doi.org/10.1111/jth.14856.

Lin, L. et al. (2020). Hypothesis for potential pathogenesis of SARS-CoV-2 infection-a review of immune changes in patients with viral pneumonia. Emerging Microbes \& Infections. 9(1), 727-732.

Makatsariya, A. et al. (2020). Thrombotic microangiopathy, DIC-syndrome and COVID-19: link with pregnancy prothrombotic state. The journal of maternalfetal \& neonatal medicine. doi: https://doi.org/10.1080/14767058.2020.1786811.

Ministério da Saúde (BR). (2002). Relatório estatístico do cadastro de coagulopatias hereditárias. Agência Nacional de Vigilância Sanitária (ANVISA). http://bvsms.saude.gov.br/bvs/publicacoes/perfil_coagulopatias_hereditarias_2002.pdf.

Mongula, J. et al. (2020). Covid-19 during pregnancy: non-reassuring fetal heart rate, placental pathology and coagulopathy. Department of Obstetrics and Gynecology, Máxima Medical Centre. doi: https://doi.org/10.1002/uog.22189. 
Moreira, A. et al. (2009). Fatores de risco associados a trombose em pacientes do estado do ceará. Revista brasileira de hematologia e hemoterapia, 132-136.

Nascimento, B., Scarpelini, S. \& Rizoli, S. (2007). Coagulopatia no trauma. Medicina (Ribeirão Preto), 40 (4), 509-517. doi: 10.11606/issn.21767262.v40i4p509-517.

Nesr, G. et al. (2020). Immune thrombocytopenia flare with mild COVID-19 infection in pregnancy: A case report. British Society for Haematology and John Wiley \& Sons Ltd. doi: https://doi.org/10.1111/bjh.16928.

Panigada, M. et al. (2020). Hypercoagulability of COVID-19 patients in intensive care unit: A report of thromboelastography findings and other parameters of hemostasis. J Thromb Haemost. https://pubmed.ncbi.nlm.nih.gov/32302438/.

Prabhu, M. et al. (2020). Pregnancy and postpartum outcomes in a universally tested population for sars-cov-2 in new york city: a prospective cohort study. An international Journal of obstetrics and gynecology - Royal College of obstetricians and gynecologists, p 1548 - 1556.

Ranucci, M. et al. (2020). The procoagulant pattern of patients with Covid-19 acute respiratory distress syndrome. J Thromb Haemost. Retrieved from: https://pubmed.ncbi.nlm.nih.gov/32302448/.

Rodrigues, C., Baia, I., Domingues, R. \& Barros, H. (2020). Pregnancy and breastfeeding during covid-19 pandemic: a systematic review of published pregnancy cases. Frontiers in public health, 8, 1-13.

Ronnie, L. et al. (2020). Complicated COVID-19 in pregnancy: a case report with severe liver and coagulation dysfunction promptly improved by delivery. BMC Pregnancy Childbirth, 20, 511. doi: https://doi.org/10.1186/s12884-020-03172-8.

Rostami, M. \& Mansouritorghabeh, H. (2020). D-dimer level in COVID-19 infection: a systematic review. Expert review of hematology, 13 (11), $1265-1275$. doi: https://doi.org/10.1080/17474086.2020.1831383 .

Saposnik, G. et al. (2011). Diagnosis and management of cerebral venous thrombosis: a statement for healthcare professionals. American Heart Association/American Stroke Association, 42 (4), 1158-92. doi: http://doi.org/10.1161/STR.0b013e31820a8364.

Servante, J. et al. (2021). Haemostatic and thrombo-embolic complications in pregnant women with COVID-19: a systematic review and critical analysis. BMC Pregnancy Childbirth, 21, 108. doi: https://doi.org/10.1186/s12884-021-03568-0.

Su, S. et al. (2016). Epidemiology, Genetic Recombination, and Pathogenesis of Coronaviruses. Trends in Microbiology, 24 (6), $490-502$.

Tang, N. et al. (2020). Abnormal coagulation parameters are associated with poor prognosis in patients with novel coronavirus pneumonia. J Thromb Haemost, 18, 844-847. doi: https://pubmed.ncbi.nlm.nih.gov/32073213/.

Vivas, D. et al. (2020). Recomendaciones sobre el tratamiento antitrombótico durante la pandemia COVID-19. Posicionamiento del Grupo de Trabajo de Trombosis Cardiovascular de la Sociedad Española de Cardiología. Revista Española de Cardiología, 73(9), $749-757$.

Wiersinga, W. et al. (2020). Pathophysiology, Transmission, Diagnosis, and Treatment of Coronavirus Disease 2019 (COVID-19). JAMA, 324(8), 782.

Wisniewski, D. \& Kluthcovsky, A. (2008). O Perfil dos Pacientes Portadores de Coagulopatias de uma Região do Sul do Brasil. Cogitare Enferm. doi: http://dx.doi.org/10.5380/ce.v13i2.12485.

Xu, Y. et al. (2020). Clinical and computed tomographic imaging features of novel coronavirus pneumonia caused by SARS-CoV-2. Journal of Infection, $80(4), 394-400$.

Yao, X. et al. (2020). Pathological evidence for residual SARS-CoV-2 in pulmonary tissues of a ready-for-discharge patient. Cell Research, $30(6), 541-543$.

Zhu, N. et al. (2020). A novel Coronavirus from patients with pneumonia in China, 2019. N Eng J Med, 1-7. doi: 10.1056/NEJMoa2001017.

Zoller, M., Irlbeck, M. \& Zwissler, B. (2020). Coronavirus Disease. Der Anaesthesist, 222-223. 